Article

\title{
Direct Observation of the M1 Transition between the Ground Term Fine Structure Levels of W VIII
}

\author{
Momoe Mita ${ }^{1}$, Hiroyuki A. Sakaue ${ }^{2}$, Daiji Kato ${ }^{2,3}$, Izumi Murakami ${ }^{2,3}$ \\ and Nobuyuki Nakamura ${ }^{1, *}$ \\ 1 Institute for Laser Science, The University of Electro-Communications, Tokyo 182-8585, Japan; \\ m_mita@ils.uec.ac.jp \\ 2 National Institute for Fusion Science, National Institutes of Natural Sciences, Toki, Gifu 509-5292, Japan; \\ sakaue@ms.nifs.ac.jp (H.A.S.); dkato@nifs.ac.jp (D.K.); mizumi@nifs.ac.jp (I.M.) \\ 3 Department of Fusion Science, SOKENDAI (The Graduate University for Advanced Studies), \\ Toki, Gifu 509-5292, Japan \\ * Correspondence: n_nakamu@ils.uec.ac.jp \\ Academic Editor: Elmar Träbert \\ Received: 1 November 2016; Accepted: 28 February 2017; Published: 8 March 2017
}

\begin{abstract}
We present a direct observation of the M1 transition between the fine structure splitting in the $4 f^{13} 5 s^{2} 5 p^{6}{ }^{2} F$ ground term of W VIII. The spectroscopic data of few-times ionized tungsten ions are important for the future ITER diagnostics, but there is a serious lack of data. The present study is part of an ongoing effort to solve this problem. Emission from the tungsten ions produced and trapped in a compact electron beam ion trap is observed with a Czerny-Turner visible spectrometer. Spectra in the EUV range are also observed at the same time to help identify the previously-unreported visible lines. The observed wavelength $574.47 \pm 0.03 \mathrm{~nm}$ (air), which corresponds to the fine structure splitting of $17,402.5 \pm 0.9 \mathrm{~cm}^{-1}$, shows reasonable agreement with the previously reported value $17,410 \pm 5 \mathrm{~cm}^{-1}$ obtained indirectly through the analysis of EUV spectra [Ryabtsev et al., Atoms 3 (2015) 273].
\end{abstract}

Keywords: EBIT; tungsten ions; visible spectroscopy; plasma diagnostics

\section{Introduction}

Tungsten will be used as a plasma-facing material in ITER, and thus is considered to be the main impurity ions in the ITER plasma [1]. In order to suppress the radiation loss due to the emission of the impurity tungsten ions, it is important to understand the influx and the charge evolution of tungsten ions in the plasma through spectroscopic diagnostics. The charge states span a wide range from neutral or $1+$ near the edge up to neonlike $(64+)$ or higher near the core $[2,3]$. There is thus a strong demand for spectroscopic data of tungsten ions for a wide range of charge states, and then for a wide range of wavelengths. In particular, it has been recently pointed out that the diagnostics and control of the edge plasma are extremely important for steady state operation of high-temperature plasmas. Thus, the atomic data of relatively low charged tungsten ions are of growing significance in the ITER plasma diagnostics [4]. However, there is a serious lack of spectroscopic data for W VIII-W XXVII as found in the data list compiled by Kramida and Shirai [5] and as also pointed out by Ralchenko [1]. Several groups have thus been recently making efforts to solve this problem. For example, Ryabtsev and co-workers [6-8] observed EUV spectra of W VIII excited in vacuum spark and made a detailed analysis of the spectra with the aid of a line identification program $[9,10]$. Through their efforts, it has been confirmed that the ground state configuration of W VIII is $4 f^{13} 5 s^{2} 5 p^{6}$, which had been uncertain in the previous experimental [11] and theoretical $[12,13]$ studies due to the competition with $4 f^{14} 5 s^{2} 5 p^{5}$. They also determined the fine structure splitting in the ground term $\left({ }^{2} F_{5 / 2}\right.$ and $\left.{ }^{2} F_{7 / 2}\right)$ to be $17,410 \pm 5 \mathrm{~cm}^{-1}[6]$. 
Low-charged tungsten ions attract attention not only from plasma physics but also from the fundamental physics point of view. Recently, visible transitions in highly charged ions are suggested as potential candidates for a precise atomic clock that can be used to test the time variation of the fundamental physical constants, such as the fine structure constant [14]. Some transitions in W VIII and W IX are named as candidates in a recent theoretical study by Berengut et al. [15]. However, the candidate transitions have yet to be experimentally identified.

We have been using an electron beam ion trap (EBIT) to observe previously unreported lines of tungsten ions [16,17]. An EBIT produces highly charged ions through successive ionization of trapped ions by a quasi-monoenergetic electron beam. It is thus possible to produce selected charge state ions by tuning the electron beam energy, and thus to obtain simple spectra which are useful to identify previously unreported lines. In this paper, we present an observation of the M1 transition between the fine structure levels of the ground term $4 f^{13} 5 s^{2} 5 p^{6}{ }^{2} F$ of W VIII. The result is compared with the previous theoretical and experimental studies.

\section{Experiment}

The experimental setup and the method of the present measurements were essentially the same as those used in our previous studies $[17,18]$. Multiply charged tungsten ions were produced with a compact electron beam ion trap (called CoBIT) at the University of Electro-Communications in Tokyo. The detailed description on the device is given in a previous paper [19]. It consists essentially of an electron gun, a drift tube, an electron collector, and a high-critical-temperature superconducting magnet. The drift tube is composed of three successive cylindrical electrodes that act as an ion trap by applying a positive potential (typically $30 \mathrm{~V}$ ) at both ends with respect to the middle electrode. The electron beam emitted from the electron gun is accelerated towards the drift tube while it is compressed by the axial magnetic field (typically $\sim 0.08 \mathrm{~T}$ ) produced by the magnet surrounding the drift tube. The compressed high-density electron beam ionizes the ions trapped in the drift tube. In the present study, tungsten was introduced into the trap through a gas injector as a vapor of $W(C O)_{6}$.

The M1 transition in W VIII, which was predicted at $574 \mathrm{~nm}$ [6], was observed with a commercial Czerny-Turner type of visible spectrometer. A biconvex lens was used to focus the emission on the entrance slit of the spectrometer. The diffracted light was detected with a liquid-nitrogen-cooled back-illuminated CCD (Princeton Instruments Spec-10:400B LN). The wavelength was calibrated using emission lines from several standard lamps placed outside CoBIT. The uncertainty of the wavelength calibration was estimated to be $0.03 \mathrm{~nm}$ including systematics.

Emission in the EUV range was also observed to help the identification of visible lines. A grazing-incidence flat-field spectrometer [20] consisting of a $1200 \mathrm{~g} / \mathrm{mm}$ concave grating (Hitachi 001-0660) and a Peltier-cooled back-illuminated CCD (Roper PIXIS-XO: 400B) was used.

\section{Results and Discussion}

Figure 1 shows the visible and EUV spectra, measured simultaneously at acceleration voltages of the electron beam of $90,100,115$, and $130 \mathrm{~V}$, as obtained from the potential difference $V_{\mathrm{cd}}$ between the cathode (electron gun) and the middle electrode of the drift tube. For simplicity, we refer to the quantity $e V_{\text {cd }}$ as "electron energy", although the actual collision energy between the beam electron and the trapped ions can be different from the $e V_{\text {cd }}$ value due to several reasons, such as the space charge of the electron beam and the trapped ions. In general, the actual electron energy is lower than the $e V_{\text {cd }}$ value by a few tens of eV due to the space charge potential of the electron beam; however, it is unsure whether it can also be applied in the present study.

In the previous study with the Livermore EBIT, the EUV spectra of tungsten ions were observed with wavelength and electron energy ranges similar to the present study, and several transitions of W VII and VIII were identified [4]. Our spectra shown in Figure 1a are generally consistent with their spectra; thus, through the comparison with their spectra, the lines of W VII and VIII can be identified as shown in the figure. For example, the line at $21.6 \mathrm{~nm}$ is assigned as the $5 p^{6}-5 p_{1 / 2}^{-1} 5 d_{3 / 2}$ transition of 
W VII. A cluster of lines near $20 \mathrm{~nm}$ mainly corresponds to the $5 p-5 d$ transitions of W VIII. It can be confirmed that the W VII lines were observed at 90 and $100 \mathrm{eV}$, and became weak at $115 \mathrm{eV}$, whereas the W VIII lines kept increasing from 90 to $115 \mathrm{eV}$ and became weak at $130 \mathrm{eV}$. Their difference in energy dependence reflects the fact that the charge distribution shifted to a higher charge as electron energy increased. Based on the energy dependence, a cluster of lines near $19 \mathrm{~nm}$ can be assigned to W IX although these were not identified in the previous Livermore spectra. According to the calculation by Ryabtsev et al. [6], a lot of transitions in W IX are predicted near 18 to $19 \mathrm{~nm}$. We consider that the cluster of lines near $19 \mathrm{~nm}$ in the present EUV spectra corresponds to a part of the predicted lines although a detailed analysis and identification are in progress. The present identification seems to be inconsistent with the ionization energy values [5] because W VIII (122 eV required) and W IX (141 eV required) lines can be found in the spectrum at an electron energy of $90 \mathrm{eV}$. Similar inconsistency is also found in the spectra of the previous Livermore spectra, where they observed W VII (65 eV required) lines at $43 \mathrm{eV}$ and $\mathrm{W}$ VIII (122 eV required) lines at $72 \mathrm{eV}$. Ionization from metastable excited states [21-23] may account for this inconsistency though we are not sure at present.
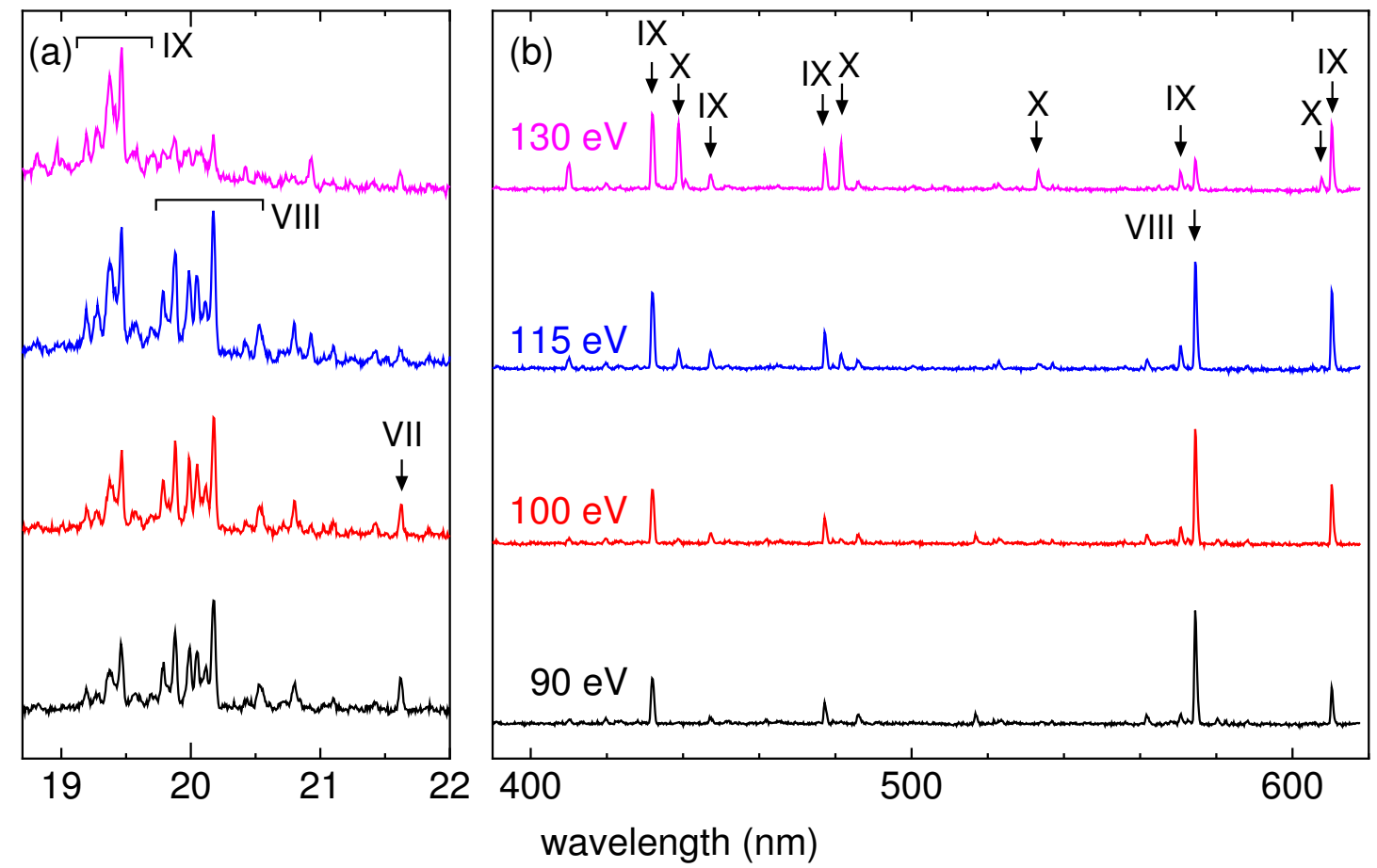

Figure 1. Spectra of tungsten ions in (a) the EUV range and (b) the visible range, obtained simultaneously at electron energies ( $e V_{\text {cd }}$, see text) of 90 (black), 100 (red), 115 (blue), and $130 \mathrm{eV}$ (pink).

In the visible spectra, the line observed at $574 \mathrm{~nm}$ shows the same electron energy dependence as the W VIII lines in the EUV spectra. Thus, it can be assigned to the transition between the fine structure splitting of the ${ }^{2} F$ ground term in W VIII. The wavelength is consistent with the energy interval 17,410 $\mathrm{cm}^{-1}$ previously obtained from the high resolution EUV spectra of vacuum sparks [6]. The visible spectra shown in Figure 1 were observed with a low dispersion grating ( $300 \mathrm{~g} / \mathrm{mm}$ blazed at $500 \mathrm{~nm}$ ) to cover a wide visible wavelength range (350 to $620 \mathrm{~nm}$ ). We have confirmed that in the observed wavelength range, the line at $574 \mathrm{~nm}$ is the only prominent line that can be assigned to W VIII. According to the calculation for W VIII by Ryabtsev et al. (see Figure 2 of Ref. [6]), the two configurations $4 f^{14} 5 p^{5}$ and $4 f^{13} 5 p^{6}$ compete for the ground state, and the other configurations have excitation energies of larger than $300,000 \mathrm{~cm}^{-1}$, which would make transitions in the EUV range. The two lowest configurations both have a doublet structure $\left({ }^{2} P_{1 / 2,3 / 2}\right.$ for $4 f^{14} 5 p^{5}$ and ${ }^{2} F_{5 / 2,7 / 2}$ for $\left.4 f^{13} 5 p^{6}\right)$, but the energy splitting for $4 f^{14} 5 p^{5} 2 P$ is too large to be observed in the visible range, therefore 
the transition between the doublet levels of $4 f^{13} 5 p^{6}{ }^{2} F$ is considered to be the only transition that falls into the visible range. This is consistent with the fact that we could observe only one prominent line that can be assigned to W VIII. It may also be possible to have more visible transitions if we can observe transitions between high excited levels. However, there is less chance to observe such transitions in the low density EBIT plasma.

In order to obtain the transition wavelength, we have also observed the visible spectrum with a higher resolution using a $1200 \mathrm{~g} / \mathrm{mm}$ grating blazed at $400 \mathrm{~nm}$. The experimental wavelength obtained from the higher resolution observation is $574.47 \pm 0.03 \mathrm{~nm}$ (air), which corresponds to a fine structure splitting of $17,402.5 \pm 0.9 \mathrm{~cm}^{-1}$. The result is listed in Table 1 together with previous values. As seen in the table, our present value shows reasonable agreement with the previous experimental value by Ryabtsev et al. [6]. It is noted that the Ryabtsev value was obtained through the analysis of the EUV spectrum containing a lot of transitions arising from highly excited states to the ground configurations. The present experiment is the first direct observation of the transition between the fine structure splitting. The present result has confirmed the reliability of the analysis done by Ryabtsev et al.

Table 1. Fine structure splitting of the ground term $4 f^{13} 5 p^{6}{ }^{2} F$ in W VIII. The uncertainty is not given in the theoretical value for Berengut [14]. The experimental value by Ryabtsev [6] was obtained indirectly through the analysis of EUV spectra.

\begin{tabular}{cccc}
\hline & Year & exp or th & Energy $\left(\mathbf{c m}^{-1}\right)$ \\
\hline Kramida [5] & 2009 & theory & $17,440 \pm 60$ \\
Berengut [14] & 2009 & theory & 18,199 \\
Ryabtsev [6] & 2015 & experiment & $17,410 \pm 5$ \\
Mita (present) & 2016 & experiment & $17,402.5 \pm 0.9$ \\
\hline
\end{tabular}

In summary, we have observed the visible M1 transition between the ground state fine structure splitting of W VIII. The energy splitting obtained from the transition wavelength has shown good agreement with the value obtained in the previous study where the energy interval was indirectly obtained from EUV spectra. We have also found previously unreported lines of W IX and W X as indicated in Figure 1, which are currently under analysis and will be published elsewhere.

Acknowledgments: This work was supported by JSPS KAKENHI Grant Numbers 16H04623, 16H04028, and partly supported by the JSPS-NRF-NSFC A3 Foresight Program in the field of Plasma Physics (NSFC: No.11261140328, NRF: No.2012K2A2A6000443). N.N. also thanks H. Tanuma for lending the CCD used in the visible spectroscopy.

Author Contributions: M.M. measured and analyzed spectra. H.A.S., D.K., and I.M. helped the analysis. N.N. supervised the work and wrote the manuscript.

Conflicts of Interest: The authors declare no conflict of interest.

\section{Abbreviations}

The following abbreviations are used in this manuscript:

EBIT Electron beam ion trap

CCD Charge Coupled Device

EUV Extreme Ultraviolet

\section{References}

1. Ralchenko, Y. Collisional-Radiative Modeling for Highly-Charged Ions of Tungsten. Plasma Fusion Res. 2013, 8, 2503024.

2. Peacock, N.J.; O'Mullane, M.G.; Barnsley, R.; Tarbutt, M. Anticipated X-ray and VUV spectroscopic data from ITER with appropriate diagnostic instrumentation. Can. J. Phys. 2008, 86, 277-284. 
3. Skinner, C.H. Applications of EBIT to magnetic fusion diagnostics. Can. J. Phys. 2008, 86, 285.

4. Clementson, J.; Lennartsson, T.; Beiersdorfer, P. Extreme ultraviolet spectra of few-times ionized tungsten for divertor plasma diagnostics. Atoms 2015, 3, 407-421.

5. Kramida, A.E.; Shirai, T. Energy levels and spectral lines of tungsten, W III through W LXXIV. At. Data Nucl. Data Tables 2009, 95, 305-474.

6. Ryabtsev, A.; Kononov, E.; Kildiyarova, R.; Tchang-Brillet, W.-Ü.L.; Wyart, J.F.; Champion, N.; Blaess, C. Spectra of W VIII and W IX in the EUV region. Atoms 2015, 3, 273-298.

7. Ryabtsev, A.; Kononov, E.Y.; Kildiyarova, R.; Tchang-Brillet, W.-Ü.L.; Wyart, J.F. $4 f^{13} 5 s^{2} 5 p^{6}-4 f^{13} 5 s^{2} 5 p^{5} 6 s$ transitions in the W VIII spectrum and spectra of isoelectronic hafnium, tantalum, and rhenium ions. Opt. Spectrosc. 2012, 113, 109-114.

8. Ryabtsev, A.; Kononov, E.Y.; Kildiyarova, R.; Tchang-Brillet, W.-Ü.L.; Wyart, J.F. The spectrum of seven times ionized tungsten (W VIII) relevant to tokamak divertor plasmas. Phys. Scr. 2013, 87, 045303.

9. Azarov, V. Formal approach to the solution of the complex-spectra identification problem. I. theory. Phys. Scr. 1991, 44, 528-538.

10. Azarov, V. Formal approach to the solution of the complex-spectra identification problem. 2. Implementation. Phys. Scr. 1993, 48, 656.

11. Veres, G.; Bakos, J.; Kardon, B. Energy levels and the vacuum ultraviolet spectrum of W VIII. J. Quant. Spectrosc. Radiat. Transf. 1996, 56, 295-301.

12. Carlson, T.A.; Nestor, C.; Wasserman, N.; McDowell, J. Calculated ionization potentials for multiply charged ions. At. Data Nucl. Data Tables 1970, 2, 63-99.

13. Sugar, J.; Kaufman, V. Seventh spectrum of tungsten (W VII); resonance lines of Hf V. Phys. Rev. A 1975, 12, 994.

14. Berengut, J.C.; Dzuba, V.A.; Flambaum, V.V. Enhanced Laboratory Sensitivity to Variation of the Fine-Structure Constant using Highly Charged Ions. Phys. Rev. Lett. 2010, 105, 120801.

15. Berengut, J.C.; Dzuba, V.A.; Flambaum, V.V.; Ong, A. Electron-Hole Transitions in Multiply Charged Ions for Precision Laser Spectroscopy and Searching for Variations in $\alpha$. Phys. Rev. Lett. 2011, 106, 210802.

16. Komatsu, A.; Sakoda, J.; Minoshima, M.; Sakaue, H.A.; Ding, X.B.; Kato, D.; Murakami, I.; Koike, F.; Nakamura, N. Visible Transitions in Highly Charged Tungsten Ions: 365-475 nm. Plasma Fusion Res. 2012, 7, 1201158.

17. Kobayashi, Y.; Kubota, K.; Omote, K.; Komatsu, A.; Sakoda, J.; Minoshima, M.; Kato, D.; Li, J.; Sakaue, H.A.; Murakami, I.; et al. Extreme ultraviolet and visible spectroscopy of promethiumlike heavy ions. Phys. Rev. A 2015, 92, 022510.

18. Kobayashi, Y.; Kato, D.; Sakaue, H.A.; Murakami, I.; Nakamura, N. Spectroscopic study of promethiumlike bismuth with an electron-beam ion trap: Search for alkali-metal-like resonance lines. Phys. Rev. A 2014, $89,010501$.

19. Nakamura, N.; Kikuchi, H.; Sakaue, H.A.; Watanabe, T. Compact electron beam ion trap for spectroscopy of moderate charge state ions. Rev. Sci. Instrum. 2008, 79, 063104.

20. Ohashi, H.; Yatsurugi, J.; Sakaue, H.A.; Nakamura, N. High resolution extreme ultraviolet spectrometer for an electron beam ion trap. Rev. Sci. Instrum. 2011, 82, 083103.

21. Träbert, E. Differential observations in spectroscopic measurements using electron beam ion traps. J. Phys. $B$ 2009, 42, 154019.

22. Sakoda, J.; Komatsu, A.; Kikuchi, H.; Nakamura, N. Visible spectroscopy of Rh-like ions. Phys. Scr. 2011, T144, 014011.

23. Windberger, A.; Torretti, F.; Borschevsky, A.; Ryabtsev, A.; Dobrodey, S.; Bekker, H.; Eliav, E.; Kaldor, U.; Ubachs, W.; Hoekstra, R.; et al. Analysis of the fine structure of $\mathrm{Sn}^{11+}-\mathrm{Sn}^{14+}$ ions by optical spectroscopy in an electron-beam ion trap. Phys. Rev. A 2016, 94, 012506.

(C) 2017 by the authors; licensee MDPI, Basel, Switzerland. This article is an open access article distributed under the terms and conditions of the Creative Commons Attribution (CC BY) license (http:/ / creativecommons.org/licenses/by/4.0/). 